\title{
IRHDF: Iris Recognition using Hybrid Domain Features
}

\author{
Arunalatha J S ${ }^{1}$, Rangaswamy $\mathrm{Y}^{2}$, Shaila $\mathrm{K}^{1}$, K B Raja ${ }^{1}$, Dinesh Anvekar ${ }^{2}$, Venugopal K R ${ }^{1}$, \\ S S Iyengar ${ }^{3}$, L M Patnaik ${ }^{4}$ \\ ${ }^{1}$ University Visvesvaraya College of Engineering, Bangalore University, Bangalore, India \\ ${ }^{2}$ Alpha College of Engineering Bangalore, India \\ ${ }^{3}$ Florida International University, Miami, Florida, USA \\ ${ }^{4}$ Indian Institute of Science, Bangalore, India \\ aruna.veeresh@yahoo.co.in, ranga.ace@gmail.com
}

\begin{abstract}
Iris Biometric is a unique physiological noninvasive trait of human beings that remains stable over a person's life. In this paper, we propose an Iris Recognition using Hybrid Domain Features (IRHDF) as Dual Tree Complex Wavelet Transform (DTCWT) and Over Lapping Local Binary Pattern (OLBP). An eye is preprocessed to extract the complex wavelet features to obtain the Region of Interest (ROI) area from an iris. OLBP is further applied on ROI to generate features of magnitude coefficients. Resultant features are generated by fusion of DTCWT and OLBP using arithmetic addition. Euclidean Distance (ED) is used to match the test iris image with database iris features to recognize a person. We observe that the values of Equal Error Rate (EER) and Total Success Rate (TSR) are better than in [7].
\end{abstract}

\section{Keywords-Biometric; DTCWT; ED; OLBP; TSR}

\section{INTRODUCTION}

Iris is a unique and distinguishing feature of an individual and thus can be used as an accurate means of biometric identification compared with other biometrics such as Fingerprint [1], Signature [2] etc., It is a colored circular part of an eye image that encircles the pupil and is encompassed by the sclera. The iris recognition system has four modules which are: (i) Acquisition of an iris image, (ii) Preprocessing, (iii) Analysis of the image pattern, and (iv) Pattern matching.

Non ideal images are still a challenge for the iris recognition systems and they directly affect the accuracy of the system based on two factors: (i) poor image quality and (ii) they cannot be pre-processed properly.

Motivation: Iris is unique, stable and is not similar even for left and right eyes of same individual; it is more accurate in biometrics. The recognition accuracy can be enhanced in iris recognition by using both frequency domain Dual Tree Complex Wavelet Transform (DTCWT) and spatial domain Over Lapping Binary Pattern (OLBP) techniques.

Contribution: In this paper, Iris Recognition using Hybrid Domain Features is proposed. The Morphological operations are used to create iris template. DTCWT and OLBP are applied to obtain iris features and ED is used for comparison.

Organization: The paper is ordered into the following sections. Section II gives a brief review of related works. Section III discusses proposed system model. Proposed algorithm of iris recognition system is presented in Section IV. Performance Analysis is explained in Section V and Conclusions are presented in Section VI.

\section{RELATED WORK}

Jaishankar et al., [3] developed kernel learning for a cross sensor Iris identification. The concept of machine learning is applied to minimize the deprivation of cross performance by adapting the samples of iris between different sensors. It has better cross-sensor recognition accuracy. It needs to address the issues of kernel dimensionality reduction and max-margin classifiers.

Chun and Ajay [4] developed an efficient and accurate at-adistance Iris recognition using geometric key-based Iris encoding for noisy iris images under less constrained environments using both visible and Mean Infrared imaging. The geometric key information is generated to encode the iris features from the localized iris region. It outperforms [5] and [6] in Equal Error Rate and Decidability index. It has higher time complexity.

Chun and Ajay [7] presented periocular and online iris recognition for relaxed imaging constraints. It develops identification of both iris and periocular region to extract both global and local features from remotely acquired iris images under less constrained conditions. It exploits random-walker algorithm efficiently to estimate coarsely segmented iris images.

Chun and Ajay [8] proposed an iris recognition method which exploits the iris features computed from both the localized iris regions and global feature representations. Zernike moments-based phase features are computed from the partially overlapping regions more effectively to accommodate local pixel region variations in the normalized iris images for stable characterization of local iris features. The proposed technique is better than [7]. 
Amol and Raghunath [9] described the extraction of iris features based on the triplet 2-dimensional bi-orthogonal wavelet using generalized Half Band Product (HBP) Filter and Triplet Half-Band Filter Bank (THFB). The THFB's provide independent parameter to change the order of regularity. The $k$-out- $n$ : A post classifier is incorporated by dividing the sub images to minimize the false rejection.

Dong et al., [5] proposed an iris matching technique using Iris training images of same class to obtain weight map. It is based on occlusion mask, local image quality, best bits and image fusion. The proposed strategy achieves enhanced iris recognition performance for poor quality iris images.

Khary et al., [10] presented the multispectral iris recognition scheme using Circular Hough Transform (CHT) and modified Local Binary Pattern (mLBP). The CHT is used to localize the iris regions from the multispectral iris images and mLBP to extract features.

\section{PROPOSED MODEL}

\section{A. Dual Tree Complex Wavelet Transform (DTCWT)}

It is an enhancement of Discrete Wavelet Transform [11] with added characteristics. It is an efficient technique to develop an analytical wavelet transform. Kingsbury [12] obtained DTCWT coefficients by using directional filters and shift invariance. The DTCWT uses two real Discrete Wavelet Transforms; the real part is the first DWT and the imaginary part is second DWT of the complex wavelet transform. Fig.1 shows the structure of DTCWT.

\section{B. Overlapping Local Binary Pattern (OLBP)}

Zhang et al., [13] introduced the LBP operator to explain textures in 2-dimensional images. The LBP characteristic features are robust even for illumination changes and are computationally simple. The LBP operator is used to label each pixel of a given image by a binary sequence using centre pixel threshold in a $3 \times 3$ matrix. The corresponding binary bits are assigned to 0 when the values of the neighboring pixels are less than the central pixel else they are assigned to 1 . The remaining eight binary bits forms a binary number and is converted into a decimal value for labeling the centre pixel of $3 \times 3$ matrix.

\section{Proposed Model}

In this part, the problem definition, goals and the proposed model are explained.

Problem Definition: The Given Iris image is used to validate the verification of a person using the combination of features of Overlapping Local Binary Pattern (OLBP) and Dual Tree Complex Wavelet Transform (DTCWT).

The goals are to:

- Increase the Total Success Rate (TSR),

- Reduce the False Acceptance Rate (FAR), False Rejection Rate (FRR) and Equal Error Rate (EER).

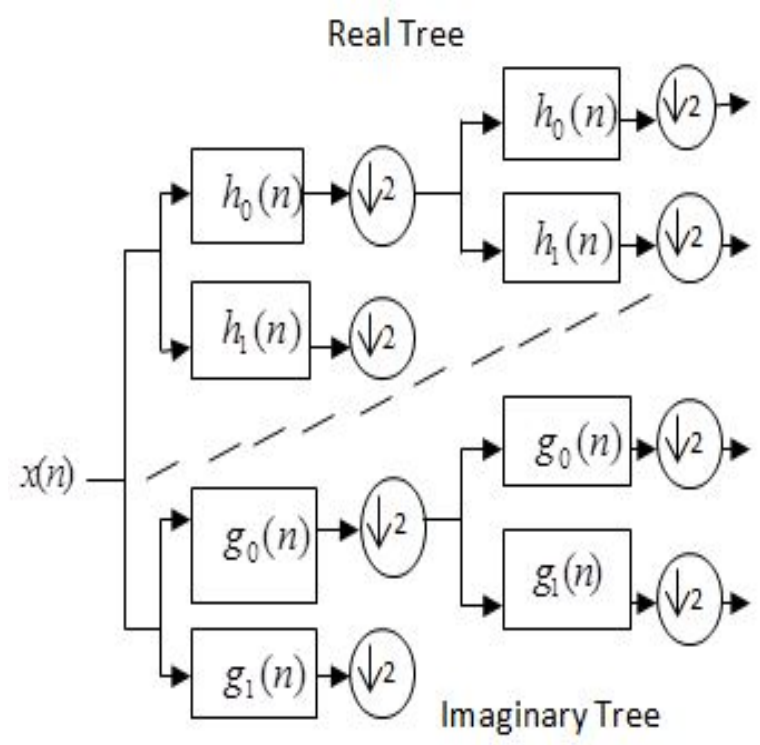

Fig. 1: Structure of DTCWT

The flow diagram of the proposed model is shown in Fig. 2.

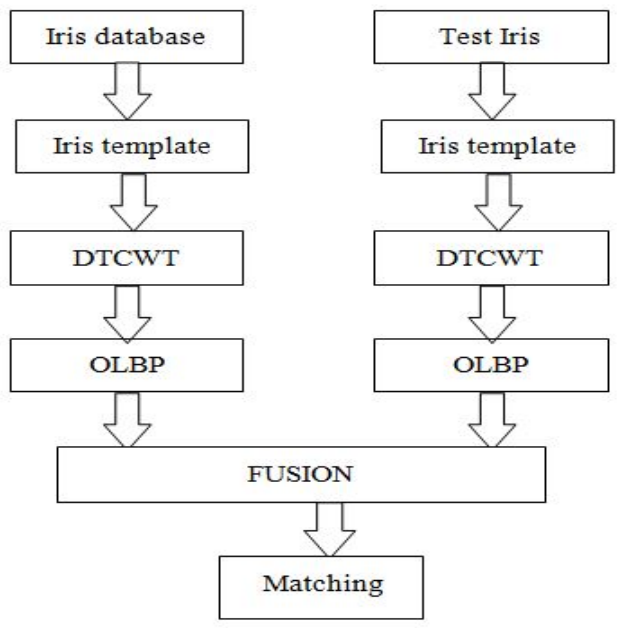

Fig. 2: Flow Diagram of the Proposed Model

\section{1) Iris database}

The database of iris is created using CASIA V1.0 (Chinese Academy of Science Institute of Automation) database [14] and [15]. It consists of grayscale images of size $280 \times 320$. CASIA V1.0 database contains seven iris images from 108 individuals. The images in the database were captured in two sessions. To mask the reflections in an iris part, all circular pupil regions in the eye images are replaced by constant intensity values which help in proper recognition of iris with accurate feature extraction with less error rate.

\section{2) Iris Template}

The Localization is performed on Eye image to select iris part located between sclera and pupil. The iris is covered by 
pupil at the inner circular boundary and sclera at the outer circular boundary. The lower and upper portions of an iris region closer to sclera are occupied by eyelashes and eyelids. The iris part surrounded by pupil is extracted by finding radius and centre of the pupil using suitable threshold. Since, its shape is simple and it is the darkest region of an eye image. Then, the Morphological procedure is applied to eliminate the eyelashes to obtain the pupil radius and center.

Pupil Detection: Connected component analysis searches the images and groups its pixels into components based on pixel connectivity. Pixels having similar intensity values are grouped and each pixel in the group is labeled. The diameter and centre of all labeled group are determined and the pupil is detected based on pixel group which has largest diameter. The eyelashes and eyelids surrounded by lower and upper portions of iris are masked by assigning all pixels below and above the diameter of the pupil as Not a Number (NaN) [16].

The input eye image is pre-processed using morphological dilation and erosion operations which uses a structuring element matrix in the form of pixel values as 1 's and 0 's and it results in different shapes for a connected component. A structuring element's origin is taken as a reference and based on neighboring values at the origin are used to dilate and erode the image. Finally, iris is localized by selecting 45 pixels from the lowest iris boundary of 80 pixels and upper boundary of 150 pixels around the pupil boundary.

\section{3) Feature Extraction}

a) Features of DTCWT: A three-stage DTCWT is used on a matrix of 64 X 64 image size. Every stage of DTCWT has 16 sub bands among twelve are high frequency and four are low frequency sub bands. The size of every $8 \times 8$ high frequency sub bands of level three is transformed into coefficient of vector size 64. The three high frequency sub-band vector coefficients of each tree are combined to obtain 192 coefficients. Then, the absolute magnitude values are computed using imaginary and real parts of trees.

The coefficient vectors of four low frequency sub bands are combined to obtain resultant low frequency Coefficients of vector size 256. In the third level, all low and high frequency coefficients are combined to obtain resultant coefficients of size 384. Then, the final coefficient vector of DTCWT is changed into matrix of size $96 \times 4$ [17].

b) Texture Features of OLBP: It is used on coefficient matrix of DTCWT by padding zeros on all parts of the matrix resulting in $98 \times 6$. It is divided into $3 \times 3$ matrix to obtain the centre coefficient value and it is assumed as reference. The neighboring values of the centre coefficient are compared with the reference coefficient. If the value of reference is less than the adjacent value of pixel coefficient then coefficient value is assigned with binary value of 1 else assigned 0 . The eight adjacent binary coefficient values are converted into decimal values and are considered as centre coefficient feature of OLBP. Similarly, feature set for all $3 \times 3$ overlapping matrix are generated by using decimal values. Finally, DTCWT is fused with OLBP to obtain the resultant coefficients [17].

\section{ALGORITHM: IRHDF}

TABLE 1: Algorithm OF IRIS RECOGNITION USING HybRID DOMAIN FEATURES (IRHDF)

Input: Image of an eye.
Output: Identification of an individual.
Step 1: Load the image of an eye.
Step 2: Creation of Iris template using Morphological
$\quad$ Operations.
Step 3: Three level Dual Tree Complex Wavelet is
$\quad$ applied on iris template.
Step 4: OLBP is used on DTCWT Features.
Step 5: Repeat steps 1 to 4 for all iris images in the
$\quad$ Database.
Step 6: Form final feature vector for each iris image.
Step 7: repeat steps 1 to 6 for the test image.
Step 8: Match and Non Match is decided by using
$\quad$ Euclidean Distance.

In the proposed algorithm of Iris Recognition using DTCWT and OLBP features, the iris database images are preprocessed using segmentation. The segmentation uses morphological dilation and erosion for creation of iris template, three levels of DTCWT is used to obtain iris template in frequency domain. Coefficients of DTCWT describe directional variations of iris images. The micro texture features like orientation, edge variations are captured by applying Overlapping Local Binary Pattern on complex wavelet coefficients which gives better recognition rate compared to traditional methods of iris recognition. The recognition rate for different number of features per image is calculated using Euclidean distance and the steps are shown in TABLE 1.

The FAR, FRR and TSR values are plotted based on the results of an algorithm. The experimental result for the value of True Success Rate has demonstrated that the proposed algorithm has better performance.

\section{PERFORMANCE ANALYSIS}

Performance analysis is based on CASIA version VI.0 database. Six image samples are considered as training images and other one is considered as test sample.

FAR, FRR plots for different threshold values are shown in Fig. 3 to Fig. 6 for different number of Persons In Database (PID) and Persons Out of Database (POD). We have considered PID: POD ratios as 20:80, 30:70, 40:60, 70:30 and 80:20. 


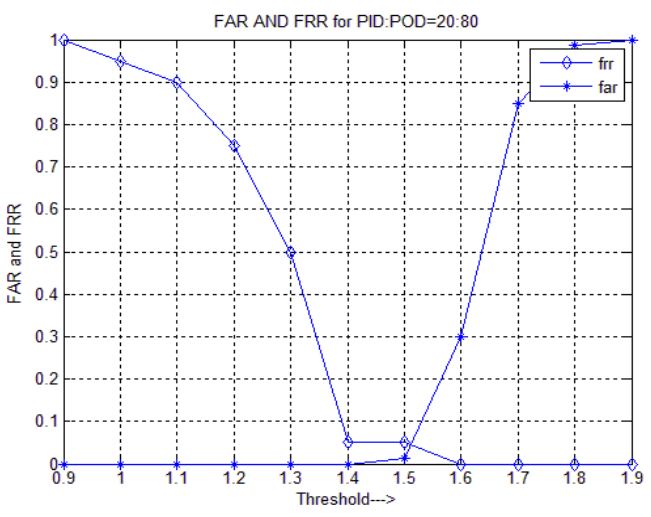

Fig. 3: FAR/FRR for PID: $P O D=20: 80$

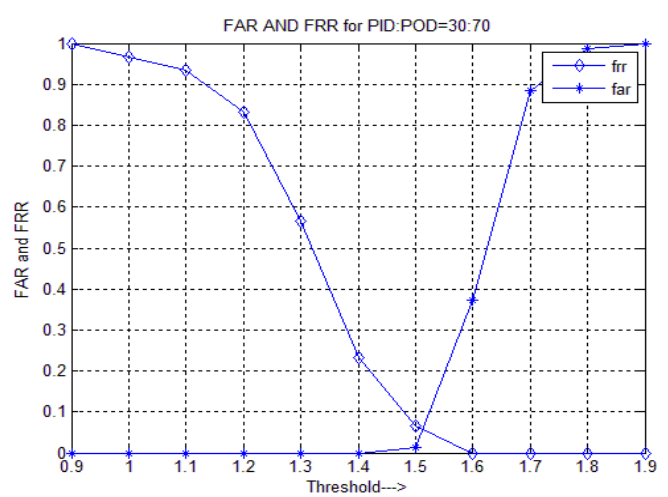

Fig. 4: FAR/FRR for PID: $P O D=30: 70$

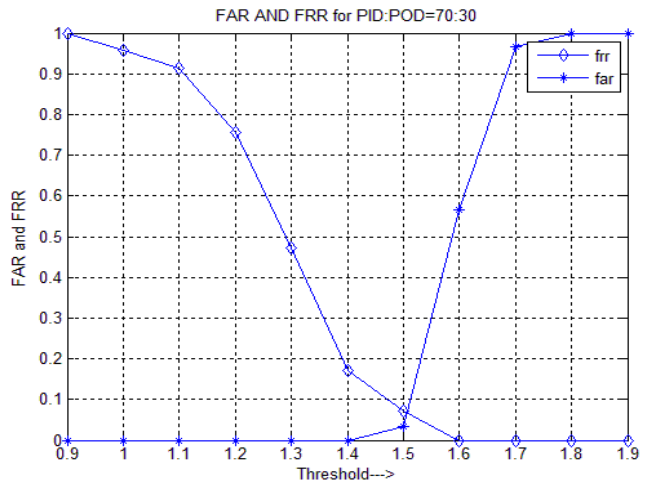

Fig. 5: FAR/FRR for PID: $P O D=70: 30$

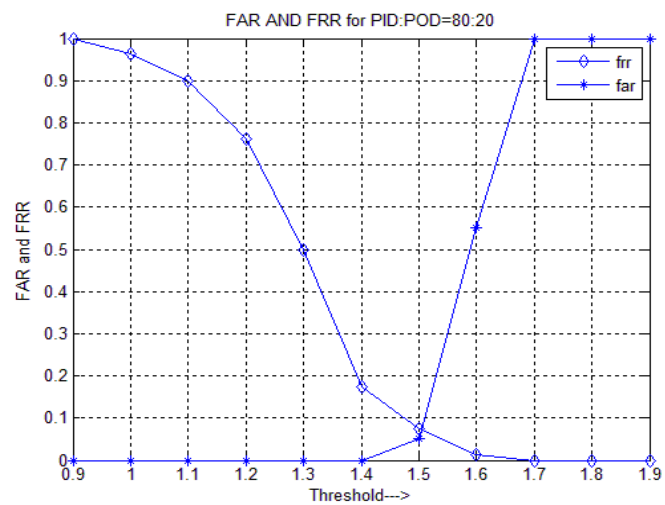

Fig. 6: FAR/FRR for PID: $P O D=80: 20$

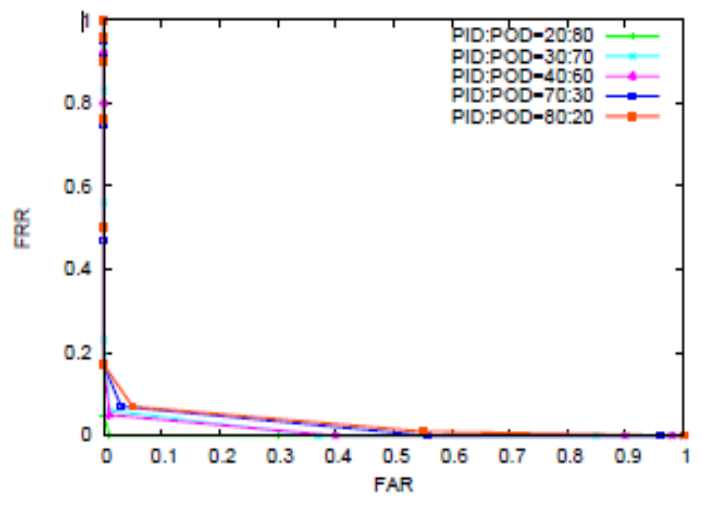

Fig. 7: ROC Characterstics

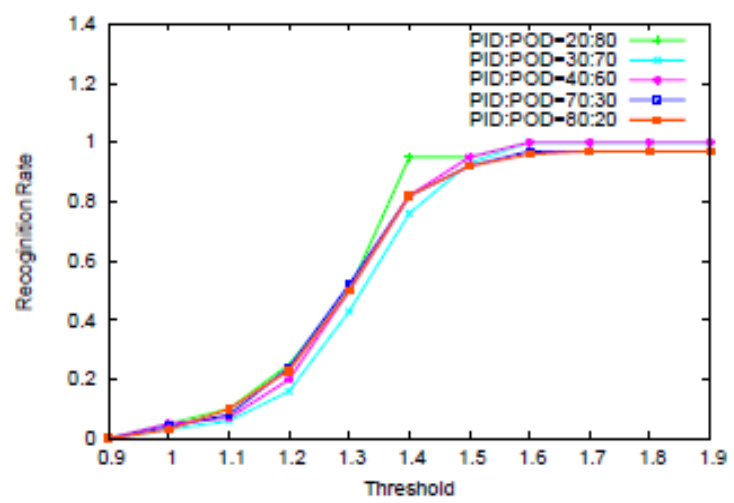

Fig. 8: Recognition Rate against Threshold

TABLE 2: COMPARISON OF TSR WITH EXISTING ALGORITHMS AND PROPOSED IRHDF

\begin{tabular}{|c|c|c|c|}
\hline $\begin{array}{c}\text { Sl. } \\
\text { No. }\end{array}$ & References & Methods & $\begin{array}{c}\text { TSR } \\
(\mathbf{\%})\end{array}$ \\
\hline 1 & Chun and Aajay [4] & $\begin{array}{c}\text { Gabor Key+Log } \\
\text { Gabor }\end{array}$ & 92.90 \\
\hline 2 & Dong et al., [5] & $\begin{array}{c}\text { Weight Map } \\
\text { feature }\end{array}$ & 95.22 \\
\hline 3 & Khary et al.,[10] & HT + MLBP & 96.00 \\
\hline 4 & Amol and Ragunath [9] & HBP+THFB & 97.96 \\
\hline 5 & Proposed $I R H D F$ & DTCWT+OLBP & 98.48 \\
\hline
\end{tabular}

The Receiver Operating Characteristics (ROC) for different PID: POD is shown Fig. 7. The ROC is plotted for FRR versus FAR for different threshold values. Fig. 8 shows variations of TSR i.e., Recognition Rate against different threshold. It is analyzed that the proposed algorithm gives enhanced performance when the number of Persons Inside Database (PID) is more compared to number of Persons Outside the Database (POD). The Proposed algorithm gives maximum recognition rate of 98.48 when the threshold is between 1.6 and 1.9. The comparison of TSR with existing algorithms and proposed IRHDF are shown in TABLE 2. 


\section{CONCLUSIONS}

The IRHDF algorithm is developed using the frequency domain DTCWT and spatial domain OLBP. The eye image is preprocessed to obtain ROI area from an iris part. The DTCWT and OLBP are applied individually on ROI to extract features. An arithmetic summation is used to generate final features from the individual features. Euclidean Distance is used to compare the iris database features with the test iris features. The IRHDF algorithm gives better TSR and EER values compared to [9] by $0.5 \%$.

\section{REFERENCES}

[1] Lavanya B. N., Raja K. B., Venugopal K. R., Patnaik L. M., "Minutiae Extraction in Fingerprint using Gabor Filter Enhancement," in proceedings of IEEE International Conference on Advances in Computing, Control \& Telecommunication Technologies, ACT '09, pp. 54 - 56, December 2009.

[2] Ramachandra, A. C., K. Pavithra, K. Yashasvini, K. B. Raja, K. R. Venugopal, and L. M. Patnaik. "Cross-Validation for Graph Matching based Offline Signature Verification." in proceedings of IEEE India Conference, INDICON 2008, vol. 1, pp. 17-22, 2008.

[3] Jaishankar K. Pillai, Maria Puertas and Rama Chellappa, "Cross-Sensor Iris Recognition through Kernel Learning", IEEE Transactions on Pattern Analysis and Machine Intelligence, vol. 36, no. 1, pp. 73-85, January 2014.

[4] Chun-Wei Tan and Ajay Kumar, "Efficient and Accurate At-a-Distance Iris Recognition using Geometric Key-Based Iris Encoding”, IEEE Transactions on Information Forensics and Security, vol. 9, no. 9, pp. 1518-1526, September 2014.

[5] W. Dong, Z. Sun, and T. Tan, "Iris Matching based on Personalized Weight Map," IEEE Transactions on Pattern Analysis and Machine Intelligence vol. 33, no. 9, pp. 1744-1757, September 2011.

[6] K. P. Hollingsworth, K. W. Bowyer, and P. J. Flynn, "The Best Bits in an Iris Code," IEEE Transactions on Pattern Analysis and Machine Intelligence, vol. 31, no. 6, pp. 964-973, June 2009.
[7] C.W. Tan and A. Kumar, "Towards Online Iris and Periocular Recognition under Relaxed Imaging Constraints," IEEE Transactions on Image Processing, vol. 22, no. 10, pp. 3751-3765, October 2013.

[8] Chun-Wei Tan and Ajay Kumar, "Accurate Iris Recognition at a Distance using Stabilized Iris Encoding and Zernike Moments Phase Features," IEEE Transactions on Image Processing, vol. 23, no. 9, pp. 3962-3974, 2014.

[9] Amol D. Rahulkar and Raghunath S. Holambe, "Half-Iris Feature Extraction and Recognition using a New Class of Biorthogonal Triplet Half-Band Filter Bank and Flexible k-out-of-n: A Post Classifier," IEEE Transactions on Information Forensics and Security, vol. 7, no.1, pp. 230-240, February 2012.

[10] Khary Popplewell, Kaushik Roy, Foysal Ahmad and Joseph Shelton, "Multispectral Iris Recognition utilizing Hough Transform and Modified LBP," in proceedings of IEEE International Conference on Systems, Man and Cybernetics, pp. 1396-1399, October 2014.

[11] H. C. Sateesh Kumar, K. B. Raja, Venugopal K. R., and L. M. Patnaik, "Automatic Image Segmentation using Wavelets," International Journal of Computer Science and Network Security, vol. 9 no.2, pp. 305-313, February 2009.

[12] Nick Kingsbury, "A Dual-Tree Complex Wavelet Transform with Improved Orthogonality and Symmetry Properties," in proceedings of International Conference on Image Processing, vol. 2, pp. 375-378, 2000 .

[13] Z. Guo, L. Zhang, and D. Zhang, "A Completed Modeling of Local Binary Pattern Operator for Texture Classification," IEEE Transactions on Image Processing, vol. 19, no. 6, pp. 1657-1663, 2010.

[14] http://www.sinobiometrics.com, CASIA Iris Image Database.

[15] http://www.springerimages.com, Springer Analysis of CASIA Database.

[16] Shashi Kumar D. R., K. B. Raja, R. K. Chhootaray, Sabyasachi Pattnaik, "PCA based Iris Recognition using DWT," International Journal on Computer Technology and Applications, vol. 2, no. 4, pp. 884-893, 2011

[17] Rangaswamy Y., Ramya S. K., K. B. Raja, Venugopal K. R., L. M. Patnaik, "Face Recognition using Transform Domain Texture Features," in proceedings of Sixth International Conference on Machine Vision (ICMV 2013), International Society for Optics and Photonics, pp. 90671P-90671P, 2013. 